\title{
Outcome of selective ramisectomy for botulinum toxin resistant torticollis
}

\author{
Blair Ford, Elan D Louis, Paul Greene, Stanley Fahn
}

\begin{abstract}
Objective-To investigate the long term outcome of selective ramisectomy denervation in patients with botulinum toxin resistant spasmodic torticollis.

Background-The published surgical series of ramisectomy treatment for torticollis do not provide systematic information on patients who develop resistance to the current standard of treatment-botulium toxin injections. Moreover, there is little information on surgical outcome using rating scale measurements of torticollis, or assessments of functional and occupational capacity.

Methods-Using a structured interview format and videotape assessments of severity of dystonia in a retrospective fashion, detailed follow up information was obtained on 16 patients who underwent open label selective denervation for severe, disabling torticollis, refractory to injections of botulinum toxin.
\end{abstract}

Results-Of 16 patients with disabling torticollis followed up postoperatively for a mean of 5 years, six (37.5\%) had a moderate or complete return of normal neck function, as determined using functional capacity scales, whereas 10 had only minimal relief of dystonia or gain in function. Six of the 16 patients $(37.5 \%)$ underwent a second peripheral denervation operation, and one required a third. Of 11 patients working outside the home before surgery, nine were disabled by dystonia, and only one continued to work after surgery. Dystonia rating scale scores of videotaped examinations using a modification of the Toronto Western Spasmodic Torticollis Rating Scale (TWSTRS) improved in 12 of 14 patients $(85.7 \%)$ who underwent selective ramisectomy. When patients with primary botulinum toxin resistance were excluded, the magnitude of benefit for this subgroup was $31.9 \%$ of the baseline dystonia score $(p<0.0002)$, comparable with the degree of improvement in a group of control patients receiving botulinum toxin treatment for torticollis.

Conclusion-About one third of patients with torticollis resistant to injections of botulinum toxin may derive modest long term functional improvement from selective denervation, with a reduction in dystonia by about $30 \%$, but remain unable to work.

(f Neurol Neurosurg Psychiatry 1998;65:472-478)

Keywords: cervical dystonia; spasmodic torticollis; denervation
Modern peripheral denervation surgery for torticollis was pioneered by Keen in $1891,{ }^{1}$ and refined over the past century as surgical techniques improved. Selective ramisectomy is the currently preferred operation, ${ }^{2}$ although no direct comparison between different surgical techniques has ever been performed. Despite many reports of therapeutic success, the long term outcome of surgical denervation is difficult to assess. In general, the surgical series do not use consistent or rigorous outcome measures, rating scales for torticollis, descriptions of functional capacity, or adequate follow up. Moreover, most surgical series were performed during an era when ramisectomy could be considered a first line treatment for torticollis due to the lack of effective alternative therapy. Since the advent of chemodenervation as the standard treatment for torticollis, an assessment of the role of peripheral surgery for patients who have become resistant to botulinum toxin is of special interest. Important issues pertaining to patient selection, prognostic factors, and functional outcome all require further study.

\section{Methods}

Between 1986 and 1995, 18 patients followed up at the Center for Dystonia, ColumbiaPresbyterian Medical Center underwent selective extradural ramisectomy procedures for severe, intractable torticollis, refractory to medications and botulinum toxin $\mathrm{A}$ injections. Patients were identified through a computerised database, and contacted for follow up assessments if they were no longer being treated at our institution. Of these 18 patients, detailed clinical information and long term follow up assessments were available on 16 patients, who form the basis for this report. Clinical information was obtained retrospectively from the patients' medical records, records of botulinum toxin injections, operative reports, and videotaped examinations. Baseline and follow up videotaped examinations were available on 14 patients. Patients had rotational torticollis, laterocollis, retrocollis, or a combination of these abnormal postures.

All patients were referred to outside institutions for surgery. The operations were performed by four surgeons at three medical centers (University of Montreal, Canada; Mayo Clinic, Rochester, Minnesota, USA; and Mayo Clinic Jacksonville, Florida, USA). For rotational torticollis, surgery consisted of a unilateral extradural rhizotomy of C1 and C2, with section of the posterior primary divisions of $\mathrm{C} 3$ to $\mathrm{C} 6$, and contralateral spinal accessory nerve 
section, as described. ${ }^{2}$ For laterocollis, the ramisectomy was ipsilateral to the sternocleidomastoid denervation. For retrocollis, the procedure was a bilateral section of cervical roots and posterior rami, $\mathrm{C} 1$ to $\mathrm{C} 5$.

Detailed follow up information was obtained using a structured interview format, conducted by telephone or in the office between 14 and 111 months after each patient's original ramisectomy, with a mean follow up of 59.5 months. We obtained retrospective descriptive information on functional and occupational capacity, medication usage, requirement for continuing botulinum toxin injections, and patients' estimates of the long term effect on neck dystonia and pain.

For the purposes of this analysis, we attempted to quantify five domains of the clinical data using simple descriptors and rating scales: activity of daily living (ADL) capacity, occupational status, medication usage, pain, and an overall functional status, estimated by the interviewer (table 1). Although patients described their condition using terms like "moderately disabled", "partly disabled", "mildly disabled", the subjective nature of these descriptions precluded a meaningful quantification of the degree of impairment. As such, we defined baseline ADL capacity simply as unimpaired, impaired, or disabled. The change in ADL capacity after surgery was estimated as worsened from baseline, unchanged, improved with residual impairment, or improved to normal. Occupational status for those working outside the home was designated as working or disabled, based on the patients' reports of attendance at work. The effect of surgery on pain was estimated as providing no benefit, pain reduction, or relief of pain. The need for medications to control dystonia or pain postoperatively was compared with baseline, taking into account the number of medications as well as daily dosages. Medication requirement postoperatively was designated as increased, unchanged, reduced, or discontinued. An overall clinical status was estimated by the interviewer using a simple four point rating scale that took into account the effect of surgery on head position and pain, the current functional and occupational capacity, and the need for ongoing treatment: $0=$ no improvement in dystonia, or worse dystonia; $1=$ mild improvement in dystonia, enabling limited gain of function; $2=$ moderate improvement in dystonia, enabling patient to function with only minor limitation or discomfort; $3=$ complete or almost complete resolution of torticollis and return of normal function.

All videotaped examinations were performed by movement disorder specialists using a routine protocol that assesses neck position during quiet sitting, neck movement in all directions, standing, and walking. The follow up videotaped examinations were made between 3 and 96 months after surgery, with a mean of 36.3 months. Standardised videotaped examinations were evaluated by two observers (BF, EL) shielded as to the surgical status of each patient's videotape segment. Ratings were performed using a modification of the TW-
STRS scale ${ }^{34}$ that we could conveniently apply to videotaped examinations (table 2). Six four point items assessing head position, range of motion, presence of tremor, and a global estimate of severity were used, as well as a six point duration rating, weighted by a factor of two. To validate this rating scale and to provide a standard of comparison for the surgical outcomes, we used videotape segments from a previous double blind, placebo controlled trial of botulinum toxin A injections ${ }^{5}$ for a comparison group. Videotaped examinations of patients before and 6 weeks after receiving botulinum toxin $\mathrm{A}$ injections were rated by the same two reviewers, blinded to treatment status, using the same rating scale. For the surgical patients, rating scale scores from the preoperative baseline examination and the most recent follow up were compared using paired $t$ tests. Videotaped examinations of the botulinum toxin control group at baseline and after treatment were also compared in this manner. The percentage change in total rating scale scores was calculated for all patients. Severity scores, duration factors, and a global severity duration rating were analysed separately. The between rater reliability was high for all items of the rating scale applied to both groups $(r=0.9048$, $\mathrm{p}<0.0001)$.

\section{Results}

The outcome of selective ramisectomy was evaluated for 16 patients with refractory torticollis, comprising five men and 11 women (table 1). The age of onset of torticollis ranged between 28 and 54 years, with a mean of 45.8 years. The patients' ages at surgery ranged between 39 and 63 years, with a mean of 52.6 years. Fifteen patients had idiopathic dystonia, and one with retrocollis had presumed tardive dystonia after metoclopramide use. All patients complained of disabling neck postures. In 14 patients, unrelenting pain was a main component of the torticollis. The primary direction of dystonia was rotational in 11 patients, laterocollis in three, and retrocollis in two, although 11 patients had combined movements. There were no patients with pure anterocollis in this series.

All patients underwent botulinum toxin A injections during the early years of treatment. Three patients never improved with botulinum toxin injections and were thus considered to have primary resistance. Twelve additional patients eventually developed clinical resistance to botulinum toxin injections, as defined by an absence of benefit and the lack of atrophy after an injection; in seven of these patients, the presence of antibodies to botulinum toxin was detected using the mouse assay. ${ }^{6}$ In 15 patients, the decision to undergo surgery was based on the development of resistance to botulinum toxin injections, and to refractory pain and torticollis. The remaining patient elected to have surgery because he could not afford the botulinum toxin injections.

According to postoperative notes and retrospective data collection, 12 patients described less dystonia and pain immediately after surgery. For 11 of these patients, this beneficial 
Table 1 Long term follow up after selective denervation for torticollis

\begin{tabular}{|c|c|c|c|c|c|c|c|}
\hline $\begin{array}{l}\text { Patient } \\
\text { No }\end{array}$ & $\begin{array}{l}\text { Age at } \\
\text { onset }\end{array}$ & $\begin{array}{l}\text { Duration of } \\
\text { follow up } \\
\text { (months) }\end{array}$ & $\begin{array}{l}\text { Primary directions } \\
\text { of torticollis }\end{array}$ & Pain & $\begin{array}{l}\text { Resistance to } \\
\text { botulinum } \\
\text { toxin }\end{array}$ & Baseline ADL capacity and effect of surgery on ADLs & $\begin{array}{l}\text { Occupational status } \\
\text { before first operation }\end{array}$ \\
\hline 1 & 36 & 84 & Rotation & $\mathrm{Y}$ & Secondary & Impaired for ADLs; no benefit & At home \\
\hline 2 & 54 & 89 & Rotation & $\mathrm{Y}$ & Secondary & Impaired for ADLs; improved & At home \\
\hline 3 & 53 & 70 & Rotation & $\mathrm{Y}$ & Primary & Disabled for ADLs; no benefit & Disabled \\
\hline 4 & 39 & 70 & Rotation & $\mathrm{Y}$ & Secondary & $\begin{array}{l}\text { Impaired for ADLs; improved with moderate residual } \\
\text { disability }\end{array}$ & At home \\
\hline 5 & 54 & 20 & Retrocollis & $\mathrm{N}$ & Primary & Impaired for ADLs; no benefit & Disabled \\
\hline 6 & 28 & 46 & Retrocollis & $\mathrm{Y}$ & Secondary & Impaired for ADLs; no benefit & Disabled \\
\hline 7 & 54 & 63 & Rotation & $\mathrm{Y}$ & - & $\begin{array}{l}\text { Impaired for ADLs; improved with mild residual } \\
\text { disability }\end{array}$ & Disabled \\
\hline 8 & 49 & 48 & Rotation & $\mathrm{Y}$ & Secondary & $\begin{array}{l}\text { Impaired for ADLs; improved with mild residual } \\
\text { disability }\end{array}$ & At home \\
\hline 9 & 36 & 111 & $\begin{array}{l}\text { Rotation and } \\
\text { laterocollis }\end{array}$ & $\mathrm{N}$ & Secondary & Impaired for ADLs; improved to normal & Working full time \\
\hline 10 & 54 & 89 & Laterocollis & $\mathrm{Y}$ & Secondary & Impaired for ADLs; no benefit & Working full time \\
\hline 11 & 41 & 76 & Rotation & $\mathrm{Y}$ & Secondary & $\begin{array}{l}\text { Disabled for ADLs; improved with mild residual } \\
\text { disability }\end{array}$ & Disabled \\
\hline 12 & 44 & 14 & $\begin{array}{l}\text { Rotation and } \\
\text { laterocollis }\end{array}$ & $\mathrm{Y}$ & Secondary & $\begin{array}{l}\text { Impaired for ADLs; improved with moderate residual } \\
\text { disability }\end{array}$ & Working full time \\
\hline 13 & 47 & 27 & Rotation & $\mathrm{Y}$ & - & $\begin{array}{l}\text { Impaired for ADLs; improved with moderate residual } \\
\text { disability }\end{array}$ & Disabled \\
\hline 14 & 50 & 75 & Rotation & $\mathrm{Y}$ & Secondary & $\begin{array}{l}\text { Impaired for ADLs; improved with mild residual } \\
\text { disability }\end{array}$ & At home \\
\hline 15 & 44 & 53 & Laterocollis & $\mathrm{Y}$ & Primary & $\begin{array}{l}\text { Impaired for ADLs; improved with moderate residual } \\
\text { disability }\end{array}$ & Disabled \\
\hline 16 & 50 & 17 & Laterocollis & $\mathrm{Y}$ & Secondary & $\begin{array}{l}\text { Impaired for ADLs; improved with moderate residual } \\
\text { disability }\end{array}$ & Disabled \\
\hline
\end{tabular}

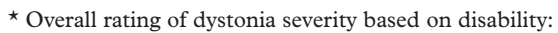

$0=$ No improvement in dystonia, or worse dystonia.

$1=$ Mild improvement in dystonia, with moderate residual disability.

$2=$ Moderate improvement in dystonia, enabling patient to function with only minor limitation or discomfort.

$3=$ Complete or almost complete resolution of torticollis and return of normal function.

ADLs=activities of daily living.

effect was not sustained, and a subsequent gradual deterioration ensued over the next several months. Two patients who derived benefit from the surgery experienced an abrupt deterioration in torticollis after minor neck trauma several months postoperatively. Two patients worsened immediately after surgery, including the patient with the worst outcome of the entire group (patient 3). After surgery, his torticollis increased greatly, and he experienced generalised spread of dystonia. $\mathrm{He}$ underwent a second peripheral denervation within six months, followed 12 days later by a stereotactic thalamotomy for intractable dystonia. It is speculative as to whether the surgery caused his dystonia to spread, but peripheral trauma is a well known precipitant of dystonia. The other patient whose torticollis worsened after surgery developed spread of the dystonia to craniofacial structures, and required additional medication to return to his preoperative state. Due to declining benefit, six of the 16 patients $(37.6 \%)$ underwent a second peripheral denervation operation, and one patient required a third. Other than worsening of dystonia in two patients, there were no significant complications reported.

After a mean follow up interval of 5.2 years, six of 16 patients had a moderate or complete return of normal neck function, designated as 2 or 3 using the functional capacity scale (table 1). Eleven patients had worked outside the home in full time professional careers when they developed torticollis. Of these 11, nine patients became disabled due to torticollis and two carried on in their work. After surgery, only one patient was able to continue working, and no patient previously disabled returned to work. Of 14 patients experiencing intractable neck pain preoperatively, eight $(62 \%)$ had sustained relief of pain after the procedures.

Patients were asked to estimate the effect of surgery relative to their most successful injections of botulinum toxin. This retrospective inquiry is subject to recall bias but is instructive because such a comparison has not been previously performed. Two patients stated that the botulinum toxin injections gave greater relief of dystonia and six stated that surgery was superior. Three patients derived equal benefit from botulinum toxin and surgery, four patients thought that the techniques were equally ineffective, and one person was unable to estimate.

Compared with their preoperative status, eight of 15 patients medically treated before surgery were taking less medication for torticollis at the end of the follow up interval, including three patients who were able to discontinue their medication. Five patients were taking the same medications in the same doses at the follow up interview, one patient was taking more medication, and one patient who took no medications before surgery was still on no medication.

Dystonia rating scale scores of videotaped examinations improved in 12 of 14 patients $(85.7 \%)$ who underwent selective ramisectomy (table 3). The magnitude of change in dystonia rating scales ranged from $+150 \%$ to $-70.3 \%$, with a mean change of $-15.4 \%$, denoting improvement. The two patients with a poorer postoperative score both had primary resistance to botulium toxin injections. The effect of one patient who deteriorated severely postoperatively was sufficient to prevent significance in the change in scores for the group as a whole (paired $t$ test $=2.15, \mathrm{p}=0.051$ ). The result from this patient could not have been predicted in 


\begin{tabular}{|c|c|c|c|c|}
\hline $\begin{array}{l}\text { Most recent } \\
\text { occupational status }\end{array}$ & Medications at follow up & Long term effect on pain & Need for more surgery & $\begin{array}{l}\text { Final overall } \\
\text { status }^{I}\end{array}$ \\
\hline At home & Same & No benefit & - & 0 \\
\hline At home & Same & Unknown & - & 1 \\
\hline Still disabled & Same & No benefit & Revision (1), thalamotomy & 0 \\
\hline At home & Reduced & Improvement; residual pain & - & 1 \\
\hline Still disabled & Increased & - & - & 0 \\
\hline Still disabled & Same & No benefit & Revisions (2) & 0 \\
\hline Retired & Reduced & Improvement; residual pain & Revision (1) & 2 \\
\hline At home & Same; still receives botulinum toxin injections & Improvement; residual pain & - & 2 \\
\hline Working full time & Reduced & - & Revision (1) & 3 \\
\hline Disabled & Same & No benefit & - & 0 \\
\hline Still disabled & Reduced & Improvement; mild residual pain & Revision (1) & 2 \\
\hline Disabled & Off meds & Complete relief & - & 2 \\
\hline Still disabled & Off meds & Improvement; residual pain & - & 1 \\
\hline At home & Reduced & Improvement; residual pain & Revision (1) & 2 \\
\hline Still disabled & Off meds & Improvement; residual pain & - & 1 \\
\hline Still disabled & - & No benefit & - & 1 \\
\hline
\end{tabular}

advance, and he is included in the analysis, despite being an outlier. If this patient were excluded from the analysis, the mean change of rating scale scores would be $-28.1 \%$, ranging from +17.4 to $-70.3(\mathrm{p}=0.0003)$. If patients with primary resistance to botulinum toxin injections were excluded from the analysis, the mean improvement in rating scale scores was $-31.9 \%(p=0.0017)$. When the severity and duration portions of the rating scale scores were analysed separately, the duration factor showed a significant improvement for the entire group $(\mathrm{p}=0.0422)$. The sample size was too small to allow identification of specific factors that could predict surgical outcome.

Videotape ratings of the comparison group of patients treated with botulinum toxin showed improvements in 13 of 14 patients $(92.8 \%)$ in modified TWSTRS scores. The scores ranged from $+5.0 \%$ to $-46 \%$, with a mean improvement of $-26.5 \%$ (paired $t$ test $=6.58, \mathrm{p}<0.0001$ ). All patients in this group had rotational torticollis or laterocollis. The mean baseline severity scores for the botulinum toxin group did not differ significantly from the baseline scores of the patients undergoing surgery (Student's $t$ test $=0.60, \mathrm{p}=0.550$ ).

\section{Discussion}

Since the development of selective denervation techniques for torticollis, numerous surgical series have been published. ${ }^{27-26}$ Many of these studies describe complete relief of dystonia or marked improvement in most patients, with reports of success ranging between $53 \%{ }^{15}$ to $88 \% .^{24}$ In most surgical series, the primary outcome measure is the patients' own reports of success, a potential source of bias in judging an unblinded procedure. The largest surgical series $^{2}$ includes 260 patients, of whom 106
(40\%) experienced an excellent result, with no detectable abnormal movements postoperatively; 124 patients (48\%) had a very good result, with only slight residual abnormalities of head posture or movements. However, as in the smaller series, the duration of follow up is not given, and it is not certain what percentage of patients had a subsequent evaluation.

Despite many reports of therapeutic success, and the demonstration that intricate denervation can be carried out safely, the many published surgical series give little information on long term outcome, disability factors, severity of dystonia based on direct examination by shielded reviewers, occupational functional capacity, need for reoperation, subsequent medical treatment, patient selection criteria, or prognostic features. Moreover, with the emergence of botulinum toxin chemodenervation as a safe and standard treatment for torticollis, an analysis of surgical denervation for patients who have undergone injections is especially warranted.

The present study, although providing a retrospective analysis of only 16 patients, attempts to consider some of the methodological problems of the surgical series. We provide detailed follow up information on 16 of 18 patients $(88.9 \%)$ who were referred from Columbia-Presbyterian Medical Center to undergo the procedures. We quantify dystonia severity using blinded ratings of videotaped examinations, and provide a comparison group of patients with dystonia of similar severity, treated by botulinum toxin injections.

Among patients with disabling torticollis resistant to botulinum toxin injections, we found a long term success rate of less than that reported in the surgical literature for selective ramisectomy, and a high incidence of 
Table 2 Torticollis rating scale: modified from TWSTRS (Consky and Lang ${ }^{4}$ )

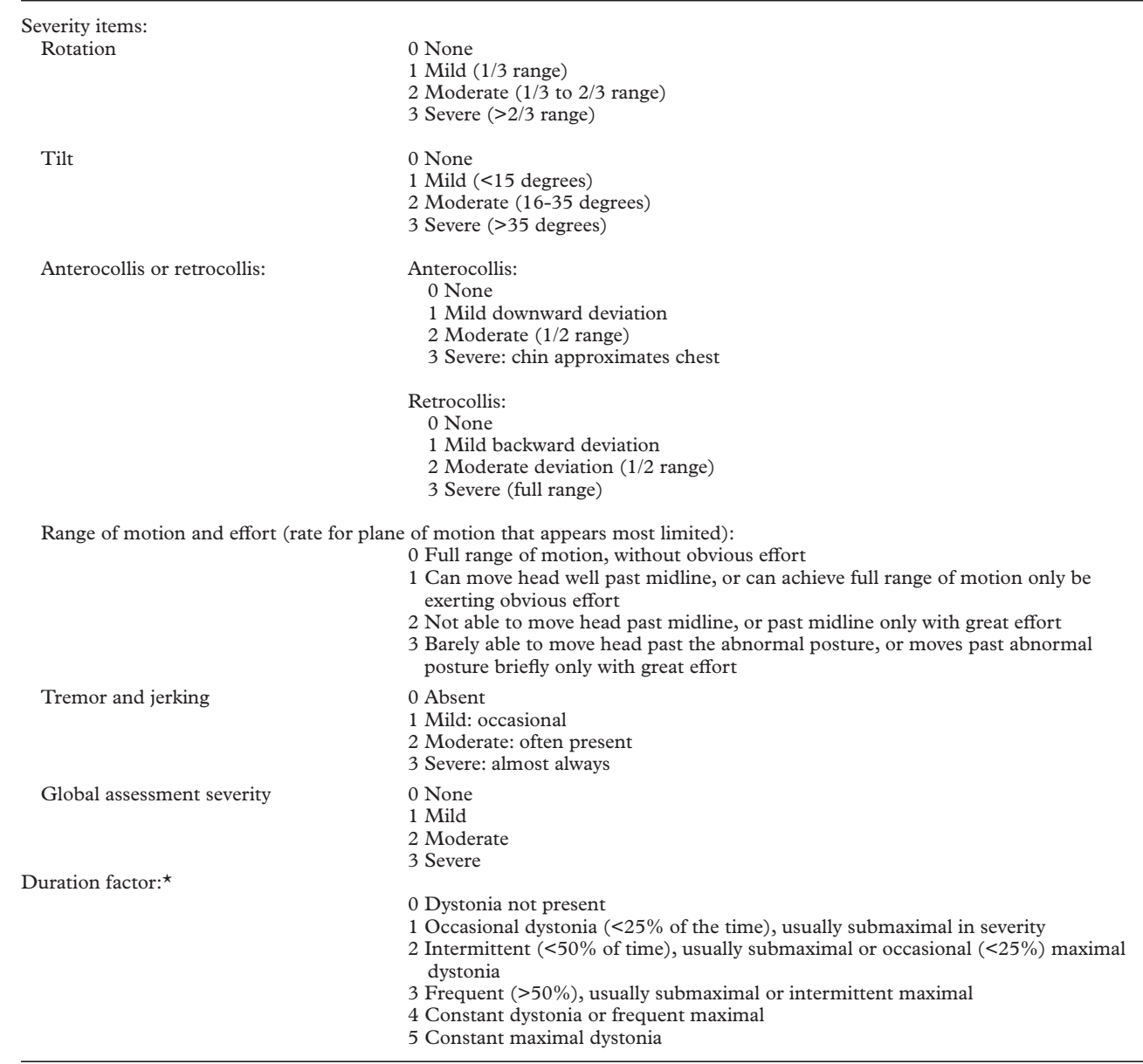

^Estimated duration of maximal dystonia as \% of time dystonia is present during each video segment. This score is weighted $\times 2$. The maximum possible total score, combining severity items and the weighted duration factor $(\times 2)$, is 28 .

reoperation. Of 16 patients followed up for a mean interval of almost 5 years, only one $(6.3 \%)$ could claim to have been cured, with a complete resolution of dystonic neck posturing, elimination of pain, and a full return to his career. Five (31.3\%) patients were moderately improved in functional capacity, but continued to require medication for dystonia or botulinum toxin injections. Five (31.3\%) patients derived only minimal relief from

Table 3 Torticollis ratings for ramisectomy patients and control patients

\begin{tabular}{|c|c|c|c|c|c|c|}
\hline & \multicolumn{3}{|c|}{ Ramisectomy group } & \multicolumn{3}{|c|}{ Botulinum toxin-control group } \\
\hline & Baseline & Follow up & Change (\%) & Baseline & Follow up & Change (\%) \\
\hline 1 & 19.25 & 18.5 & -3.9 & 15.75 & 9.25 & -41.3 \\
\hline 2 & 11.25 & 8.25 & -26.7 & 9.75 & 9 & -7.7 \\
\hline 3 & 8.5 & 21.25 & +150.0 & 19 & 16.75 & -11.8 \\
\hline 4 & 14 & 11.5 & -17.9 & 12.5 & 6.75 & -46.0 \\
\hline 5 & 5.75 & 6.75 & +17.4 & 19.25 & 14.25 & -26.0 \\
\hline 6 & 21.75 & 19.25 & -11.5 & 15 & 8.75 & -41.7 \\
\hline 7 & 16 & 4.75 & -70.3 & 9.25 & 6.75 & -27.0 \\
\hline 8 & 18.75 & 9.75 & -48.0 & 5 & 5.25 & +5.0 \\
\hline 9 & 8.25 & 4.25 & -48.5 & 14 & 11 & -21.4 \\
\hline 10 & 19.75 & 15 & -24.1 & 18 & 14.75 & -18.0 \\
\hline 11 & 16.75 & 12.5 & -25.4 & 12.75 & 8 & -37.2 \\
\hline 12 & 14 & 9 & -35.7 & 12.25 & 10 & -18.4 \\
\hline 13 & 17 & 11 & -35.3 & 13.5 & 7 & -48.1 \\
\hline 14 & 15.25 & 10 & -34.4 & 15.25 & 10.5 & -31.1 \\
\hline Mean & 14.7 & 11.6 & -15.3 & 13.7 & 9.9 & -26.5 \\
\hline SD & 4.8 & 5.3 & 52.1 & 3.9 & 3.4 & 15.6 \\
\hline
\end{tabular}

Torticollis ratings are a modification of the TWSTRS (see table 2). The percentage change in score refers to the baseline score. A negative change in the modified TWSTRS score, meaning a reduction in score, represents a lessening of torticollis severity; a positive change denotes an increase.

All ratings are the mean of two blinded observers $(r=0.9048, \mathrm{p}<0.0001)$.

Mean change in dystonia scores for ramisectomy patients $15.3 \%$ (paired $t$ test $=2.15, \mathrm{p}=0.051$ ).

Mean change in dystonia scores for botulinum toxin patients $26.5 \%$ (paired $t$ test $=6.58, \mathrm{p}<0.0001$ ).

Baseline dystonia scores, comparing ramisectomy patients with botulinum toxin patients, showed no significant difference (Student's $t$ test $=0.60, \mathrm{p}=0.550)$. 
torticollis, four $(25 \%)$ were unchanged, and one $(6.3 \%)$ had progression and generalisation of dystonia. Although patients may experience improvements in neck position that can be seen on videotaped examinations, the degree of functional improvement is rarely sufficient to reverse an occupational disability. Of 11 patients working outside the home and disabled from their careers, only one patient had returned to full time work at the follow up interval.

Review of videotaped examinations by blinded observers provides an objective measure of surgical outcome. Rating scales of severity were determined at a mean follow up of 36.3 months for 14 patients, of whom 12 $(86 \%)$ showed an improvement in head position after selective denervation. The degree of improvement for the group as a whole was small, approaching $15 \%(\mathrm{p}=0.05)$. When those with primary resistance to botulinum toxin injections were excluded, the degree of improvement was about $30 \%$, comparable with the degree of change in a double blind, placebo controlled study of botulinum toxin. ${ }^{5}$ The use of rating scales to assess videotaped examinations can remove bias from the assessment, but does not measure subjective elements such as pain and disability. Although 12 of $14(87.5 \%)$ patients were improved judging by videotape segment ratings, only six (of 16 patients overall, $37.5 \%$ ) had important functional gain. As such, even significant improvements in dystonia rating scale scores do not necessarily translate into functional benefit, underscoring the need to use functional scales in assessing the outcome of a treatment for a disabling condition.

Our results indicate that most patients undergoing selective ramisectomy may anticipate a mild to moderate degree of improvement, but that the excellent results and cures often encountered in the surgical literature are more elusive. The potential reasons for this discrepancy are several, and could include the longer duration of follow up in the present study and the emphasis on functional outcome measures. Most surgical series emphasise patients' reports of satisfaction with the procedure, a potential source of outcome bias. ${ }^{16}$ Many patients reported an initial benefit after surgery, and this would have resulted in a better outcome had the follow up interval been shorter. It is also possible that patients we referred for surgery had more severe dystonia of longer duration than the patients in the surgical series, many of whom underwent selective denervation as a primary treatment, before the widespread use of botulinum toxin.

Botulinum toxin chemodenervation has been the treatment of choice for torticollis because of its superior efficacy to placebo in double blind, placebo controlled trials. ${ }^{5}$ However, patients who receive repeated injections of botulinum toxin may develop resistance to this therapy. ${ }^{67}$ Resistance to chemodenervation was the primary reason for surgical referral in the present study, and this subgroup of patients represents a therapeutic challenge. As more patients develop resistance to the strains of botulinum toxin, surgical intervention will be increasingly considered.

The relative effects of surgery and botulinum toxin have not been studied to date. In the present study, we evaluated videotaped examinations of patients treated with botulinum toxin A injections as a control for the surgical group. The magnitude of change in dystonia rating scale severity scores was comparable for botulinum toxin injections and surgical denervation. In theory, surgery offers a more extensive denervation than is possible using injections of botulinum toxin, even when guided by EMG. As such, we have regarded surgery as a last treatment resort for patients with intractable or botulinum toxin resistant torticollis. The patients who underwent ramisectomy in the present series were asked to retrospectively compare the effect of surgery with their best response to a botulinum toxin injection but a direct comparison of surgical denervation and botulinum toxin injections has never been made, and requires a prospective design.

The reasons for the failure of extensive surgical denervation have been speculated to include inadequate postoperative physical therapy, sprouting of severed nerve roots, and re-innervation of deeper, inaccessible muscles. ${ }^{28}$ With long term follow up, it is possible that the dystonia itself becomes more severe or widespread, ${ }^{29}{ }^{30}$ as happened to one patient. Also, complicating factors such as the development of cervical spine disease may begin to contribute to the pain and disability of patients with torticollis. In our population, no patient underwent cervical spine operations, but systematic data on cervical disc disease or signs of root or spinal cord compression were not collected. For many patients, torticollis represents an unrelenting pain syndrome, which can be complicated by the depression that in turn contributes to disability.

What are the indications for surgery in patients with torticollis? Which factors predict a better outcome? Within the limitations of a retrospective study, we may conclude that selective ramisectomy is a useful treatment for selected patients with torticollis that is refractory to botulinum toxin injections. It remains difficult to predict in advance which patients are most likely to experience benefit. Due to sample size limitations, we could not identify significant clinical predictors of surgical outcome. However, we may generalise, based on our experience, that the best candidates for surgery are those patients with rotational or laterocollis, who responded to botulinum toxin injections at least once. Most patients may expect a benefit equal to their most successful response to botulinum toxin, and an improvement in dystonia rating scale scores of about $30 \%$ is to be anticipated. Patients with primary resistance to injections are unlikely to derive significant benefit from surgery, and patients with retrocollis are unlikely to improve. 
We were greatly assisted in gathering follow up information by Drs Reina Benabou, Robert Burke, John Rogers, and especially Claude Bertrand, who generously provided preoperative videotaped segments on several patients. We are also grateful to those patients who returned to the Neurological Institute for interviews and videotaping. EDL is supported by federal grant NIH NS01863 and the Paul Beeson Physician Faculty Scholars in Aging Research Award.

1 Keen WW. New operation for spasmodic wry neck: namely, division or exsection of the nerves supplying the posterio rotator muscles of the head. Ann Surg 1891;13:44.

2 Bertrand C. Selective denervation for torticollis. Surg Neurol 1993;40:96-103.

3 Consky E, Basinski A, Belle L, et al. The Toronto Western spasmodic torticollis rating scale (TWSTRS). Neurology 1990;40(suppl 1):445

4 Consky ES, Lang AE. Clinical assessments of patients with cervical dystonia. In: Jankovic J, Hallett $M$, eds. Therapy with botulinum toxin. New York: Marcel Dekker, 1994.21137 .

5 Greene P, Kang U, Fahn S, et al. Double-blind, placebocontrolled trial of botulinum toxin injections for the treatment of spasmodic torticollis. Neurology 1990;40:1213-18.

ment of spasmodic torticollis. Neurology 1990;40:1213-18.
6 Zuber M, Sebald M, Bathien N, et al. Botulinum antibodies in dystonic patients treated with type A botulinum toxin: in dystonic patients treated with type A botulinum toxin:
frequency and significance. Neurology 1993;43:1715-8.

frequency and significance. Neurology 1993;43:1715-8.
7 Finney JMT, Hughson W. Spasmodic torticollis. Ann Surg Finney JMT, Hug

8 Dandy WE. An operation for the treatment of spasmodic torticollis. Arch Surg 1930;20:1021-32.

9 Patterson RM, Little SC. Spasmodic torticollis. $\mathcal{F}$ Nerv Ment Dis 1943;98:571.

10 Putnam TJ, Herz E, Glazer GH. Spasmodic torticollis III surgical treatment. Archives of Neurology and Psychiatry 1949;61:240-7.

11 Poppen JL, Martinez-Niochet A. Spasmodic torticollis. Surg Clin N Amer 1951;31:883-90.

12 McKenzie KG. The surgical treatment of spasmodic torticollis. Clin Neurosurg 1955;2:37-43.

13 Bunts AT. The surgical treatment of spasmodic torticollis. Am Surg 1960;25:560-3.

14 Sorenson BF, Hamby WB. Spasmodic torticollis: results in 71 treated patients. Neurology 1966;16:867-78.
15 Hamby WB, Schiffer S. Spasmodic torticollis: results after rhizotomy in 50 cases. 7 Neurosurgery 1969;31:323-6.

16 Meares R. Natural history of spasmodic torticollis and effect of surgery. Lancet 1971;ii:149-51

17 Arseni C, Maretis M. The surgical treatment of spasmodic torticollis. Neurochirugica 1971; 14:177-80.

18 Tasker RR. The treatment of spasmodic torticollis by peripheral denervation: the McKenzie operation. In: Morley T, ed. Current controversies in neurosurgery. Philadelphia: Saunders, 1976:448-54.

19 Chen XK. Selective resection and denervation of cervical muscles in the treatment of spasmodic torticollis: results in 60 cases. Neurosurgery 1981;8:680-8.

20 Gauthier S, Perot P, Bertrand G. Role of surgical anterior rhizotomies in the managment of spasmodic torticollis. Adv Neurol 1988:50:633-6.

21 Hernesniemi J, Keranen T. Long-term outcome after surgery for spasmodic torticollis. Acta Neurochir (Wien) 1990;103:128-30.

22 Davis HD, Ahlskog JE, Litchy WJ, et al. Selective peripheral denervation for torticollis: preliminary results. Mayo Clin Proc 1991;66:365-71.

23 Braun V, Richter H-P. Selective peripheral denervation in patients with spasmodic torticollis. Neurosurgery 1994;35: 58-62.

24 Arce C, Russo L. Selective peripheral denervation: a surgical alternative in the treatment for spasmodic torticollis. Review of 55 patients. Mov Disord 1992;7(suppl 1):128.

25 Friedman AH, Nashold BS, Sharp R, et al. Treatment of spasmodic torticollis with intradural selective rhizotomies. f Neurosurg 1993;78:46-53.

26 Krauss JK, Toups EG, Jankovic J, et al. Symptomatic and Krauss JK, Toups EG, Jankovic J, et al. Symptomatic and nia. F Neurol Neurosurg Psychiatry 1997;63:642-8.

27 Greene P, Fahn S, Diamond B. Development of resistance to botulinum toxin type A in patients with torticollis. Mov Disord 1994;9:213-7.

28 Braun V. Selective denervation for spasmodic torticollis: is the outcome always predictable. F Neurol 1995;242:504-7.

29 Lowenstein DH, Aminoff MJ. The clinical course of spasmodic torticollis. Neurology 1988;38:530-3.

30 Greene P, Kang UJ, Fahn S. Spread of symptoms in idiopathic torsion dystonia. Mov Disord 1995;10:143-52. 